\title{
Hepatitis C in Deutschland:
}

\section{Eine Analyse von Daten gesetzlicher Krankenkassen}

\author{
Jörg Tomeczkowski ${ }^{1}$, Markus Cornberg ${ }^{2}$
}

\section{Zusammenfassung}

Hintergrund und Fragestellung I Anhand von Krankenkassendaten sollte die Prävalenz von Hepatitis-C-Virus-Infektion (HCV) und Leistungen der gesetzlichen Krankenversicherung (GKV) ermittelt werden. Außerdem sollte untersucht werden wie die Spezialisierung des behandelnden Arztes die Diagnosequalität, Therapie, Verordnungsdauer und Einhaltung von Viruslastkontrollen beeinflusst.

Patienten und Methodik | Es wurden Datenbanken mit mehreren Millionen Versicherten aus den Jahren 2007 bis 2011 von jeweils bundesweit agierenden gesetzlichen Krankenkassen ausgewertet.

Ergebnisse I Bei dauerhaft Versicherten lag der Anteil der Patienten mit HCV in einem 3-JahresZeitraum bei $0,19 \% .19 \%$ der Patienten erhielten die Erstdiagnose „akute Hepatitis C“. Die jährliche Therapiequote (pegyliertes Interferon alpha mit und ohne Ribavirin) lag bei 9,3\% (11,9\% bei chronischer und 3,6\% bei akuter Hepatitis C), wobei Hausärzte 5,1\% und Internisten 21,7\% ihrer HCV-Patienten therapierten. Von Hausärzten erhielten im 3. Quartal nach Therapiebeginn noch $37,0 \%$ und von Internisten noch 57,0\% der Patienten Verordnungen. Viruslastkontrollen waren nicht in allen Fällen nachweisbar.

Folgerungen | Ca. 50\% der in Deutschland vermuteten Personen mit HCV-Infektion suchten in einem 3-Jahres-Zeitraum einen Arzt auf. Ca. 9\% dieser Personen lösten innerhalb eines Jahres Rezepte für eine HCV-Therapie ein. Bei der Kodierung einer akuten HCV-Diagnose, der Verordnungsdauer und den für die Therapie notwendigen Laborkontrollen zeigten die Analysen Abweichungen von den Empfehlungen der Leitlinie und hierbei Unterschiede zwischen Haus- und Fachärzten. Neuere Therapien sollten, um ihre Effizienz auch entfalten können, in spezialisierten Zentren durchgeführt werden.

\section{Einleitung}

Die Hepatitis C wird durch das Hepatitis-C-Virus (HCV) ausgelöst. Die akute HCV-Infektion verläuft überwiegend symptomarm und geht in 50-85\% der Fälle in eine chronische Infektion über [12]. Die chronische HCV-Infektion ist eine der häufigsten Ursachen für chronische Lebererkrankungen $[3,9,14]$ und notwendige Lebertransplantationen in Europa [14]. Unbehandelt führt sie zur Leberzirrhose mit einem hohen Risiko für ein hepatozelluläres Karzinom (HCC).

Die hohe genetische Variabilität des HCV (7 Genotypen mit bis zu 67 Subtypen [15]) erschwert die Entwicklung von Schutzimpfungen und von kausalen, medikamentösen Therapien. Bislang basierte die Standardtherapie auf Interferon. Interferon alpha wurde als Monotherapie eingesetzt. Pegyliertes Interferon wurde sowohl alleine als auch in Kombination mit Ribavirin verwendet [12] (im Folgenden als pegInterferon/Ribavirin bezeichnet). Im Jahr 2011 wurden Telaprevir (IN$\mathrm{CIVO}^{\circledR}$ ) und Boceprevir (Victrelis ${ }^{\circledR}$ ) als neue direkt antivirale Medikamente (DAM) gegen das HCV Genotyp 1 zugelassen [5, 7]. Telaprevir und Boceprevir sind HCV-Proteasehemmer, die in Kombination mit pegInterferon / Ribavirin gegeben wer- den müssen. Die interferonbasierte Therapie ist nicht für alle Patienten geeignet [6]. Seit dem Jahr 2014 sind mit Sofosbovir (Sovaldi ${ }^{\circledR}$ ), Simeprevir $\left(\right.$ Olysio $^{\circledR}$ ), Ledipasvir/Sofosbovir (Harvoni ${ }^{\circledR}$ ), Daclatasvir (Daklinza ${ }^{\circledR}$ ), Paritaprevir/r, Ombitasvir $\left(\right.$ Viekirax $^{\circledR}$ ) und Dasabuvir (Exviera ${ }^{\circledR}$ ) [4] weitere DAM zugelassen worden, die in Kombination ohne Interferon eingesetzt werden können, so dass wir einen Paradigmenwechel in der Therapie der chronischen Hepatitis C mit hohen Heilungsraten erleben werden [2]. Daher wird es umso wichtiger, möglichst viele Patienten effizient zu behandeln, bevor es bei ihnen zu Leberzirrhosen oder hepatozellulären Karzinomen kommt [18].

HCV-Infektionen müssen an das Robert Koch-Institut (RKI) gemeldet werden. In die Statistik werden alle neu diagnostizierten HCV-Infektionen aufgenommen [9]. Dennoch ist die Primärdatenlage zur Epidemiologie und Versorgungssituation der Hepatitis C in Deutschland generell unbefriedigend. Das RKI gibt eine Prävalenz in Höhe von $0,4 \%$ an [9]. Bei Blutspendern liegt der Wert deutlich niedriger [8], während groß angelegte Untersuchungen in Notfallambulanzen eine Prävalenz deutlich oberhalb von 0,6\% melden [16]. Zudem muss von einer hohen Dunkelziffer ausgegangen werden. Als ergänzende Datenquelle bieten sich
Institute

1 Janssen-Cilag $\mathrm{GmbH}$ Abteilung Health Economics, Neuss

2 Klinik für Gastroenterologie, Hepatologie und Endokrinologie, Medizinische Hochschule Hannover

\section{Korrespondenz}

Dr. rer. nat. Jörg Tomeczkowski Janssen-Cilag $\mathrm{GmbH}$ Abteilung Health Economics Johnson \& Johnson Platz 1 41470 Neuss 02137/955-384

jtomeczk@its.jnj.com 
Sekundärdaten, d. h. Krankenkassendaten, an. Mit ihnen lässt sich beurteilen, ob die an sich effektiven Therapien die Patienten erreichen und sie im Gesundheitssystem effizient eingesetzt werden.

Das Ziel dieser Arbeit war es Krankenkassendaten auszuwerten und die Anzahl Personen zu ermitteln, die Leistungen der gesetzlichen Krankenversicherung (GKV) wegen einer HCV-Infektion erhalten haben. Es wurden demografische Daten wie Alter und Geschlecht untersucht. Des Weiteren wurden Daten zur Therapie, Verordnungsdauer und Einhaltung der Viruslastkontrollen bei Behandlung ermittelt und in Abhängigkeit von der Spezialisierung der Ärzte ausgewertet.

\section{Methodik}

Es wurden Datenbanken mit unterschiedlichen Schwerpunkten ausgewertet ( Tab. 1).

- Data-to-Decision (D-to-D): 20 Krankenkassen

- Team Gesundheit GmbH (TG): Betriebskrankenkassen.

Beide Datenbanken basieren auf den Daten von verschiedenen bundesweit agierenden und nichtfusionierten gesetzlichen Krankenkassen $(\checkmark$ Tab. 1). Die Patientenzusammensetzung von D-to-D entsprach in der Alters- und Geschlechtsverteilung den Patienten aller gesetzlicher Kran-

Abb. 1 A) Prozentuale Verteilung der HCV-Infizierten in Sekundärdaten im Jahre 2008 (D-to-D) $(n=2628)$. B) Prävalenz der HCV-Infizierten innerhalb der Altersklassen in Sekundärdaten im Jahr 2010 (TG) (n=3127).
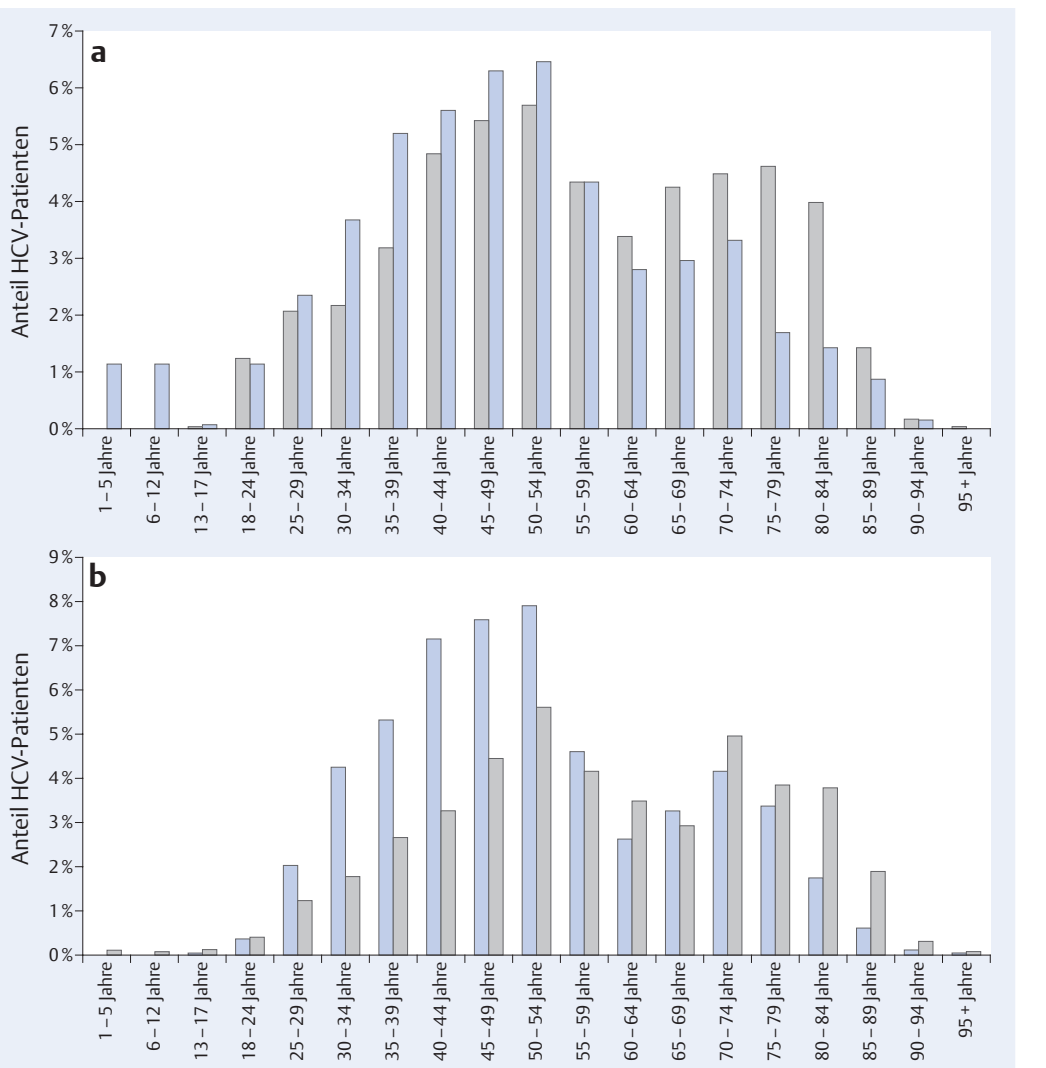

kenkassen (GKV). In der Stichprobe von TG waren jüngere Versicherte stärker vertreten als in der GKV-Gesamtheit: besonders die Anteile der Altersgruppen von 20 bis 44 Jahren wichen bis $\mathrm{zu}$ $3 \%$ ab. Der Frauenanteil war mit ca. 52\% im Vergleich zur GKV mit 53\% geringer.

In den Datenbanken wurden Personen mit mindestens einer ambulant oder stationär gesicherten HCV-Infektion (Kürzel „G“) anhand des ICD10-WHO-Kodes identifiziert:

- B17.1: akute HCV-Infektion

- B18.2: chronische HCV-Infektion.

Die Therapiedauer wurde nur für Patienten ermittelt, deren erste Verordnung von Ribavirin und pegInterferon frühestens aus dem ersten Quartal und spätestens aus dem vierten Quartal des Jahres 2008 stammte. Für die ausgewählten Patienten wurde sichergestellt, dass 12 Monate vor der ersten Verordnung keine andere Interferon-Verschreibung vorlag. Für alle Patienten wurden die 4 Quartale nach der ersten Verordnung bis ins Jahr 2009 ausgewertet. Fehlte in einem Quartal nach Therapiebeginn eine Verschreibung, gingen wir von einem Therapie-Bruch aus.

Bei den Laborkontrollen und der Anzahl Verordnungen wurde analog verfahren. Bei den behandelnden Ärzte wurden zwischen „Hausärzten“ und „Internisten“ unterschieden. Als Hausärzte galten:

- praktischer Arzt

\section{- Arzt oder Facharzt für Allgemeinmedizin}

Zur Gruppe der „Internisten“ zählten die Ärzte für:

- Innere Medizin (Hausarzt)

- Innere Medizin

- Gastroenterologie

- Angiologie

- Endokrinologie und Diabetologie

- Hämatologie und Onkologie

- Kardiologie

- Nephrologie

- Pneumologie

- Innere Medizin/Rheumatologie

\section{Ergebnisse}

Prävalenz | In den Krankenkassendaten mit dauerhaft Versicherten lag der Anteil der HCV-infizierten Patienten (akut und chronisch) innerhalb eines Jahres im Mittel bei $0,17 \%$ und innerhalb von 3 Jahren bei 0,19\% ( Tab. 1).

Die Häufigkeit der HCV-Infektion war altersabhängig ( $\bullet$ Abb. 1). Die meisten Erkrankten gab es in den Altersgruppen zwischen 25 und 59 Jahren, dabei überwog der Männeranteil. In den Altersgruppen zwischen 60 und 90 Jahren war der Anteil der weiblichen Patienten höher. 


\begin{tabular}{|c|c|c|c|}
\hline Datenquelle & D-to-D & TG & TG \\
\hline Jahr & 2008 & 2007-2009 & 2010 \\
\hline Versicherte mit & $N=1193464(\%)$ & $N=1770532(\%)$ & $N=2500195$ (\%) \\
\hline HCV Diagnose n & $2628(0,22)$ & $3281(0,19)$ & $3127(0,13)$ \\
\hline Davon akut & $791(0,07)$ & $1618(0,09)$ & $716(0,03)$ \\
\hline Davon chronisch & $1837(0,15)$ & $2456(0,14)$ & $2411(0,10)$ \\
\hline Davon akut und chronisch & n.u. & $793(0,04)$ & n.u. \\
\hline
\end{tabular}

D-to-D = Data to Decision; TG = Team Gesundheit: $\mathrm{n}$. u. = nicht untersucht

Diagnose und Therapie I In den Krankenkassendaten waren in den Jahren 2007-2009 bei bis zu $39,7 \%$ der HCV-Patienten mindestens eine Diagnose als akute HCV-Infektion (B17.1) kodiert worden. Bei 22,7\% der Patienten war ausschließlich eine Diagnose der akuten Hepatitis C verzeichnet.

Im Jahr 2010 wurde durch eine Antikörperbestimmung bei 358 Patienten die HCV-Diagnose gestellt (entspricht $11,5 \%$ aller HCV-Diagnosen im Jahr 2010). 19,1\% dieser Patienten erhielten die Erstdiagnose einer akuten Hepatitis C. Die restlichen Patienten hatten eine chronische Hepatitis. Bei 80,1\% der Patienten war die Diagnose der akuten Hepatitis auch im 4. Quartal nach Diagnosestellung noch nachweisbar. In 57,6\% der Fälle wurde diese Diagnose von Allgemeinmedizinern und in 33,2\% der Fälle von Internisten gestellt ( Abb. 2).

Bei 9\% der Patienten wurde die Diagnose Hepatitis $C$ ausschließlich im Krankenhaus kodiert. Die Diagnose war weder vor noch nach dem Krankenhausaufenthalt im ambulanten Bereich nachweisbar.

In einem 3-Jahres-Zeitraum (2007-2009) erhielten $17,6 \%$ der Patienten eine Therapie (unabhängig vom Zeitpunkt und Ort der Diagnosestellung). Im Jahr 2008 alleine waren es 9,3\% (11,9\% bei chronischer und 3,6\% bei akuter Hepatitis $C$ ). Insgesamt war die absolute Therapiequote bei Internisten mit $21,7 \%$ deutlich höher als die bei Hausärzten mit 5,1\%.

In den Kassendaten unterschieden sich Hausärzte und Internisten hinsichtlich der Anteile an Patienten mit akuter und chronischer HCV-Infektion und hinsichtlich der Therapiequoten $(\triangleright$ Abb. 2 ).

Laut Kassendaten erhielten im Jahr 2010 von den 716 Patienten mit ausschließlich akuter Hepatitis C 15 (2,1\%) eine Therapie. 2 Patienten erhielten eine Monotherapie mit Ribavirin und 13 eine Kombinationstherapie mit Ribavirin und pegInterferon. Von den 2411 Patienten mit der Diagnose einer chronischen Hepatitis C erhielten 265 (11,0\%) eine Therapie. 7 wurden mit Ribavirin, 4 mit pegInterferon und 254 mit der Kombination von Ribavirin und pegInterferon behandelt.
Laborkontrollen I Bei 132 Patienten konnte ermittelt werden, wie häufig eine Viruslastbestimmung (RNA) zur Therapienotwendigkeit und Therapiekontrolle abgerechnet wurde ( Abb. 3). Dabei wurden die Zeiträume 12 Monate vor (im Jahr 2009/2010) und 12 Monate nach Therapiebeginn (im Jahr 2010/2011) betrachtet. Von den Patienten wurden 128 mit pegInterferon/Ribavirin und 4 mit Ribavirin behandelt.

Eine Genotyp-Bestimmung wurde bei 50\% der Patienten innerhalb eines Jahres vor oder während der Therapie festgehalten. Die RNA-Bestimmung wurde am häufigsten während der Therapie abgerechnet $(60 \%$ vor und $75 \%$ nach Therapiebeginn) ( Abb. 3). Am meisten rechneten Internisten die RNA-Bestimmung vor und während der Therapie ab ( Abb. 4).

Therapiedauer I In den Kassendaten wurde ermittelt, wie häufig nach Therapiebeginn mit pegInterferon/Ribavirin weitere von Verordnungen von Internisten oder Hausärzten durch die Patienten eingelöst wurden und wie schlüssig die Verordnungen für eine chronische Hepatitis waren. Als schlüssig wurden nur die Verordnungen eingestuft, bei denen jeweils beide Wirkstoffe (pegInterferon und Ribavirin) zum notwendigen Zeitpunkt verschieben wurden und bei denen kein Bruch der Therapie durch einen Arztwechsel erfolgte. Dabei wurde festgestellt, dass bei Hausärzten in 49,1\% der Fälle und bei Internisten in 33,6\% der Fälle die Verordnungen nicht schlüssig waren. Zum Teil wurde nur einer der Wirkstoffe ver-

Abb. 2 Relative Anteile der Patienten mit akuter Hepatitis C, mit chronischer Hepatitis C und Patienten unter einer antiviralen Therapie bei Hausärzten, Internisten und anderen Arztgruppen (TG 2010) (chronische HCV n=2905; akute HCV n= 804; Therapie $n=267$ ).

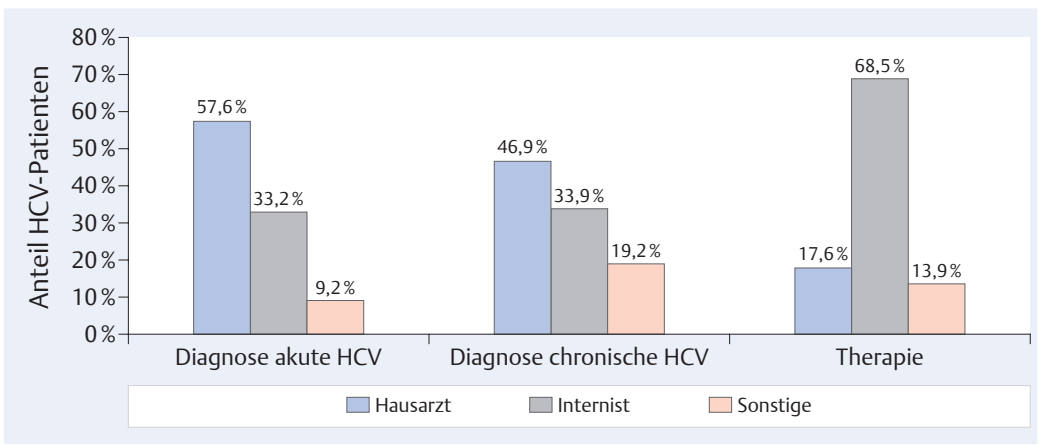

Tab. 1 Analyseparameter und Häufigkeiten der HCV Diagnosen in den Datenbanken 


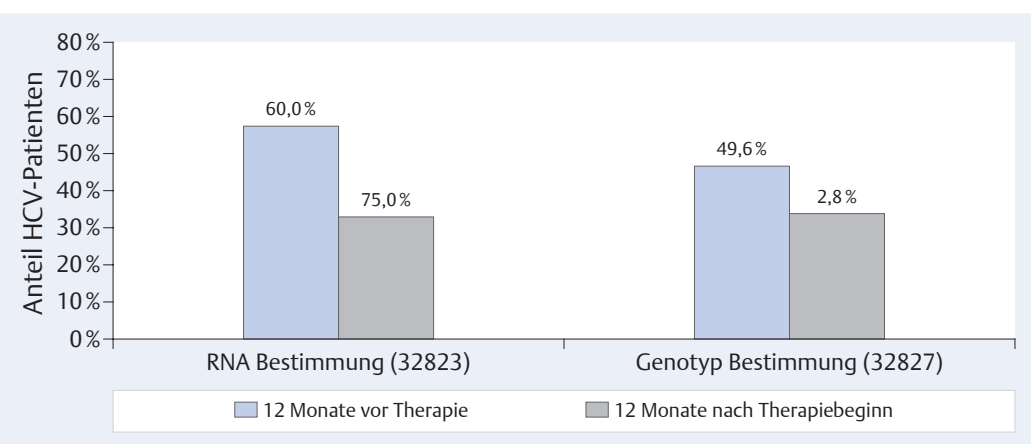

Abb. 3 Anteil der Patienten mit Abrechnung der RNA-Bestimmung (Viruslastbestimmung) und Genotyp-Bestimmung 12 Monate vor und nach Therapiebeginn bei chronischer Hepatitis C (Patienten mit Therapie $n=132$, Datenanalyse TG 2010, Beobachtungszeitraum 2009-2011). Abrechnungsziffern des Einheitlichen Bemessungsmaßstabes (EBM) in Klammern.

schrieben, wie es für die Therapie einer akuten Hepatitis üblich ist. Diese „nicht-schlüssigen“ Verschreibungen wurden von der Analyse zur Verordnungsdauer über 5 Quartale ausgeschlossen. $40 \%$ der internistisch und $33 \%$ der hausärztlich betreuten Patienten lösten die Rezepte durchgehend über 4 Quartale ein. ( Abb. 5).

In den Daten von TG (Jahr 2010) wurde bei 128 Patienten mit Kombinationstherapie (4 mit akuter und 122 mit chronischer Hepatitis C Diagnose) die durchschnittliche Anzahl Verordnungen pro Facharztgruppe ermittelt. Internisten verordneten im Durchschnitt 6 mal pegInterferon und 7 mal Ribavirin pro Patient, während Hausärzte durchschnittlich $4 \mathrm{x}$ pegInterferon und $10 \mathrm{x}$ Ribavirin verschrieben.

\section{Diskussion}

Die chronische Hepatitis $C$ wird durch die rasante Entwicklung der direkt antiviralen Therapien aktuell in den Fokus gerückt. Heilungsraten von über 90\% sind seit 2015 für die meisten Patientengruppen möglich [2]. Die Effizienz der Therapie hängt allerdings nicht nur von der Effek-

Abb. 4 Abbildung 4: Relative Anteile der anfordernden Arztgruppe für Viruslastbestimmungen (RNA-Test) in den individuellen 12 Monaten vor und nach Therapiebeginn (TG 2010, Beobachtungszeitraum 2009-2011) (Patienten mit akuter oder chronischer HCV-Diagnose und Therapie $\mathrm{n}=132$; RNA vor Therapie $\mathrm{n}=129$; RNA nach Therapie $\mathrm{n}=357$ ).

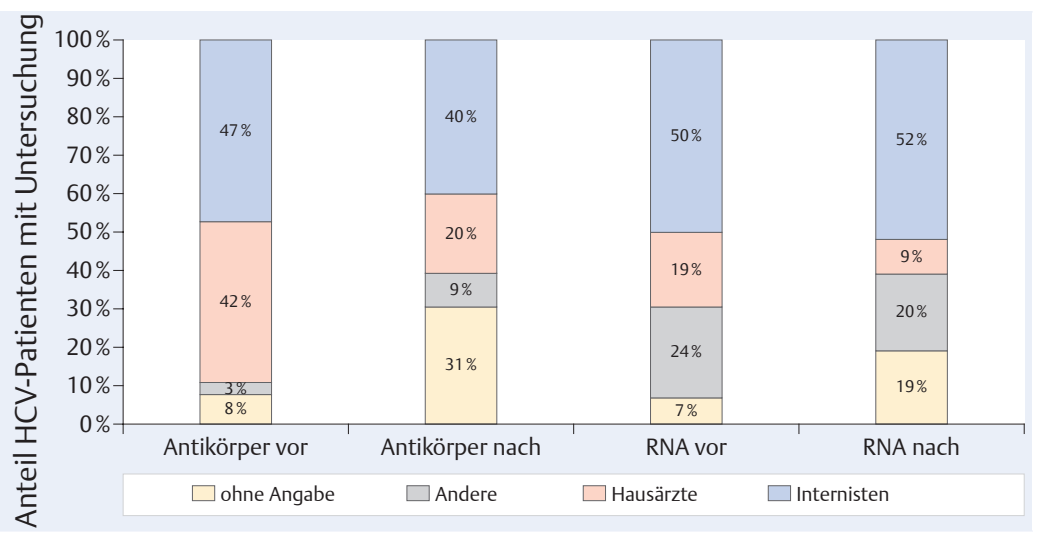

tivität der Therapie sondern auch von der Anzahl der diagnostizierten und korrekt behandelten Patienten ab [18].

In den Krankenkassendaten betrug die 1-JahresPrävalenz von HCV-Infektion im Mittel 0,17\% und die 3-Jahres-Prävalenz lag bei 0,19\%. Damit ist die Prävalenz in Sekundärdaten unter dem Wert des RKIs von 0,4\% [9]. Die untersuchten Datenquellen und die Quellen des RKI enthalten unterschiedliche Personengruppen und sind deshalb nicht direkt miteinander vergleichbar. So ist davon auszugehen, dass einige HCV-Infizierte nicht in Erwerbsbiografien eingestiegen sind und daher nicht in einer der hier untersuchten Krankenkassen versichert waren. Die Prävalenz würde damit unterschätzt werden. Die Daten vom RKI wurden im Rahmen des Bundes-Gesundheitssurveys 1998 aus einer Stichprobe von 6748 Teilnehmern im Alter zwischen 18 und 79 Jahren ermittelt. Dabei betrug die Prävalenz von Anti-HCV in der deutschen Bevölkerung 0,4\% (95\%-Konfidenzintervall 0,2-0,5\%). Die tatsächliche HCV-Antikörper-Prävalenz dürfte allerdings höher liegen. Personen aus Krankenhäusern und Justizvollzugsanstalten waren von diesen Studien ausgeschlossen. Auch waren Risikogruppen, wie intravenös Drogenabhängige, sicherlich nicht repräsentativ vertreten. Die Angaben aus den Krankenkassendaten stammen von Personen jeden Alters, die wegen einer Hepatitis-C-Virusinfektion in den Jahren 2007-2010 einen Arzt aufgesucht hatten. Personen, die entweder mit bekannter oder unbekannter HCV-Infektion nicht in diesem Zeitraum wegen HCV beim Arzt waren, wurden also auch nicht erfasst. Bei beiden Datenquellen bilden also unterschiedliche Personengruppen den Nenner (Krankenkassendaten = kranke und gesunde Versicherte, Erhebungen des RKI = selektive Bevölkerungsgruppen) und keine der Datenquellen ist repräsentativ für die Prävalenz der HCV-Infektion in der Gesamtbevölkerung.

Die Prävalenz der HCV-Infektion in Abrechnungsdaten (Sekundärdaten) war niedriger als die Werte aus gezielt vorgenommenen Reihenuntersuchungen (Notfällen oder der Stichprobe im Bundesgesundheitssurvey). Diese Diskrepanz könnte darauf hinweisen, dass in Deutschland nur jeder zweite HCV-Infizierte Leistungen der GKV in Anspruch nahm (Untersuchungszeitraum von 3 Jahren). Bei ca. $20 \%$ HCV-Infizierten wurde die Infektion erst durch eine Reihenuntersuchung bekannt [16]. Diese Personen konnten vor ihrer Diagnose auch keine Leistungen der gesetzlichen Krankenversicherung wegen einer Hepatitis in Anspruch nehmen. Ein weiterer Grund für niedrigere Prävalenz in Krankenkassendaten könnte sein, dass möglicherweise gerade Personengruppen mit erhöhter Prävalenz nicht gesetzlich krankenversichert waren (illegale Migranten, Drogenkonsumenten, Gefängnisinsassen). Ferner wurden die ICD-Kodes B17.9 (Akute Virushepatitis nicht nä- 
her bezeichnet) und B18.9 (Chronische Virushepatitis nicht näher bezeichnet) nicht untersucht.

Die ausgewerteten Daten zeigen, dass in der Altersgruppe zwischen 25 und 59 Jahren der Anteil der Männer und in den Altersgruppen zwischen 60 und 90 Jahren der Anteil der Frauen überwog. Dies ist vermutlich auf unterschiedliche Transmissionswege zurückzuführen. Bei intravenös injizierenden Drogenkonsumenten liegt der Durchseuchungsgrad mit HCV zwischen $60 \%$ und $80 \%$. Da bei Jüngeren der Drogenkonsum am höchsten ist, sind sie auch mittlerweile stärker von der HCV-Infektion betroffen. Auch das RKI gibt für das Jahr 2010 an, dass vor allem jüngere Patienten im Alter zwischen 25 und 39 Jahren neu an einer HCV-Infektion erkrankt sind und unter diesen der Anteil der Männer deutlich höher war [9].

Ein großer Anteil der HCV-Infektionen geht auf Bluttransfusionen und Blutprodukte vor dem Jahr 1990 zurück. 80-90\% der Hämophilie-Patienten, die vor dem Jahr 1987 Blutgerinnungsfaktoren erhalten haben, weisen eine HCV-Infektion auf [14]. Sekundär- und Versorgungsdaten enthalten nur Patienten, die wegen der HCV-Infektion beim Arzt waren, während die Daten des RKI alle gemeldeten Fälle (auch wenn sie im Rahmen anderer Untersuchungen oder Behandlungen festgestellt wurden) und alle Prävalenz-Schätzungen aus Reihenuntersuchungen enthalten. Insofern kann es durchaus sein, dass zwar die Gruppe der älteren Frauen mit HCV-Infektion in Deutschland überwiegt, dass aber mehrheitlich jüngere Männer Leistungen der GKV aufgrund eines schlechteren Allgemeinzustandes bei HCV-Infektion in Anspruch nehmen. Die überproportionale Inanspruchnahme von Leistungen durch Frauen im Alter 60-90 könnte darauf zurückzuführen sein, dass es bei Frauen zu einer schnelleren Fibroseprogression nach der Menopause kommt [17].

Patienten mit Verdacht auf eine HCV-Infektion werden häufig nicht zu einem Spezialisten überwiesen, sondern zunächst vom Hausarzt untersucht. Dieser bestimmt HCV-Antikörper mithilfe eines Immuntests $[1,12]$. In den Daten der Krankenkassen konnten wir ermitteln, dass die Diagnose „akute HCV-Infektion“ am häufigsten von Hausärzten gestellt wurde. Die Diagnose „akute HCV-Infektion " kann möglicherweise auch bei einer chronischen HCV-Infektion gestellt worden sein, wenn die HCV-Infektion zum ersten Mal festgestellt wurde und der Infektionszeitpunkt nicht bekannt war. Insofern könnte das gefundene unterschiedliche Verhältnis von akuten und chronischen HCV-Infektions-Diagnosen bei Haus- und Fachärzten auch die Häufigkeit von Erst- und Folgediagnosen widerspiegeln und nicht das echte Verhältnis von akuten und chronischen HCV-Infektionen. In den Kassendaten konnten wir auch feststellen, dass ca. 80\% der Patienten mit der Neu-Diagnose einer akuten Hepatitis C diese

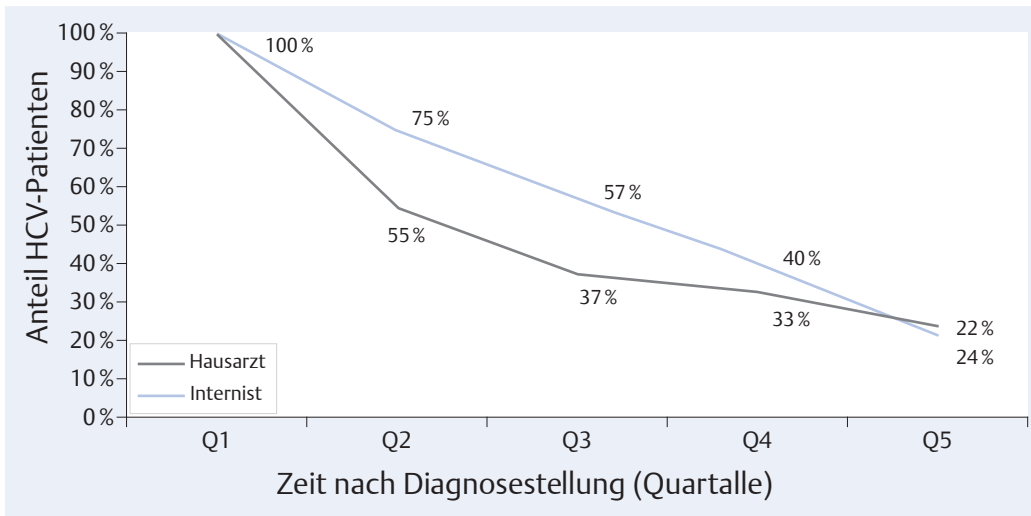

Abb. 5 Anteil der Patienten mit akuter oder chronischer Hepatitis C mit eingelösten Verordnungen für peglnterferon / Ribavirin pro Quartal (Q) nach Therapiebeginn in Krankenkassendaten. Datenanalyse D-to-D (SGH Consulting) ( $n=245)$.

Diagnose auch 9 Monate später noch aufweisen. Nach Definition der Leitlinie [12] geht aber spätestens nach 6 Monaten eine akute Hepatitis $C$ in eine chronische Hepatitis $C$ über, so dass davon auszugehen ist, dass in den Kassendaten Patienten mit akuter Hepatitis C Diagnose kumulieren.

Die Diagnose Hepatitis C wurde relativ gesehen häufiger von Hausärzten gestellt, die Behandlung allerdings wurde häufiger durch einen Spezialisten durchgeführt. Während nur 5,1\% der mit HCV infizierten Patienten bei einem Hausarzt therapiert wurden, waren es $21,7 \%$ der Patienten beim Internisten. Dies könnte auf den komplexen Therapieverlauf und die vielen notwendigen Kontrollen bei der Anwendung von antiviralen Medikamenten zurückzuführen sein. Auch die Häufigkeit der Folgeverordnungen nach Therapiebeginn und die Zusammensetzung der Kombinationstherapie war abhängig von der behandelnden Facharztgruppe: nach Therapiebeginn erhielten die Patienten von Internisten prozentual mehr Folgeverordnungen und häufiger pegInterferon als die hausärztlich betreuten Patienten. Entweder waren die Aufklärungen der Patienten und die Maßnahmen zur Therapietreue bei Spezialisten und Hausärzten unterschiedlich oder Hausärzte behandeln häufiger akute HCV-Infektionen und chronische HCV-Infektionen vom Genotyp 2/3. In diesem Fall reicht eine Therapie bis zu 24 Wochen in der Regel aus [12]. Eine andere Erklärung könnte sein, dass Patienten bei kompliziertem Therapieverlauf an Schwerpunktpraxen überwiesen werden. Die Unterschiede bei der Verordnungshäufigkeit und Zusammensetzung der Kombinationstherapie können nicht alleine durch die Therapie akuter HCV-Infektionen erklärt werden, da der Anteil dieser Patienten nur ca. $3 \%$ betrug.

2007-2011, im Erhebungszeitraum der Studie, betrug die leitliniengerechte Standardbehandlungsdauer von Patienten mit chronischer HCVInfektion in der Regel 48 Wochen für Genotyp 1 und jeweils 24 Wochen für die Genotypen 2 und 
3 [12, 13]. Bei Bedarf konnte die Therapie auch auf bis zu 72 Wochen ausgeweitet werden [12]. Therapiedauer und Dosis sollten jeweils individuell an den Gesundheitszustand des Patienten angepasst werden. Ein Therapieabbruch nach 12 Wochen bei fehlendem Abfall der HCV-RNA wurde empfohlen. Bei Patienten mit niedrigeren HCV-RNA-Werten $(<800000 \mathrm{IE} / \mathrm{ml})$ und schnellem Ansprechen (sogenannte RVR) war eine Verkürzung der Behandlungsdauer möglich [12].

Die Therapiedauer, gemessen anhand der Verordnungen, betrug in unseren Analysen bei ca. 50\% der Patienten nur 24 Wochen. Dafür kommen neben den o.g. Punkten weitere verschiedene Gründe in Betracht. Viele Patienten kommen aus sozialen Randgruppen, wodurch die Mitarbeit bei einer Therapie nicht immer gegeben ist. HCV-Infizierte haben oft Begleiterkrankungen, wie zum Beispiel eine HIV-Infektion, die eine Behandlung von HCV erschweren [12]. Die Therapie mit pegInterferon/Ribavirin wird häufig aufgrund ihrer starken Nebenwirkungen abgebrochen, insbesondere bei Ausbleiben des Therapieerfolgs [12].

Ferner konnte gezeigt werden, dass die für die Therapie notwendigen Laborverlaufskontrollen nicht immer abgerechnet werden. Ohne diese Verlaufskontrollen ist eine Verkürzung der Therapie nicht leitliniengerecht. Die vorliegenden Untersuchungen deuten darauf hin, dass Hausärzte seltener die Kombinationstherapie verordneten als Internisten und weniger Rezepte für pegInterferon dafür mehr für Ribavirin ausstellten. Einen Unterschied gab es auch bei den, für eine Therapieverkürzung notwendigen, Viruslastkontrollen (RNA-Test). Während bei den Internisten ca. 50\% der abgerechneten Viruslastkontrollen vor und während der Therapie durchgeführt wurden, waren es bei den Hausärzten 19\% vor und nur $9 \%$ während der Therapie. Somit erscheint ein unterschiedliches Patientenkollektiv bei Haus- und Fachärzten (zum Beispiel Patienten mit Genotyp 1 versus Patienten mit Genotyp 2/3) als alleinige Erklärung für die Unterschiede in der Verordnungsdauer nicht ausreichend, da auch bei Patienten mit Genotyp 2/3 vor einer Therapieverkürzung RNA-Kontrollen erfolgen sollten.

Weiterer Verbesserungsbedarf besteht möglicherweise auch bei den 9\% der HCV-Patienten, deren Diagnose ausschließlich im Krankenhaus kodiert wurde und nicht in den Patientenakten der ambulant tätigen Ärzte verschlüsselt worden war. Diese Patienten erhalten anscheinend wegen dieser HCV-Diagnose keine Nachsorge im ambulanten Bereich.

Limitationen I In Krankenkassendaten werden keine klinischen Daten erfasst. Insofern kann die Plausibilität der gemachten Angaben zur Abrechnung nicht überprüft werden. $\mathrm{Zu}$ beachten ist, dass diese Daten nicht zum Zweck der Versor- gungsforschung, sondern zum Zweck der Abrechnungsbegründung erhoben werden. Anhand dieser Daten lässt sich also nicht nachweisen, ob eine Leistung nicht erbracht wurde. Genauso wenig lässt sich feststellen, ob eine Leistung erbracht aber nicht abgerechnet wurde. Bei den vorliegenden Auswertungen ist zu beachten, dass eine, im Rahmen von klinischen Studien durchgeführte, Therapie nicht in den ausgewerteten Daten der Krankenkassen erscheint, wodurch die Therapiequote unterschätzt werden kann.

\section{Fazit}

Die neueren direkt antiviralen Substanzen werden die Effektivität der Hepatitis-C-Therapie weiter steigern. Diese neuen Therapien werden zunächst sehr teuer sein. Die Überwachung der Therapie und der unerwünschten Arzneimittelwirkungen erfordert spezialisierte Kenntnisse [10,11]. Die hier gezeigten Daten zur geringen Therapierate, dauerhaften Kodierung der Diagnose akute Hepatitis C, verkürzten Verordnungsdauer und geringen Rate an Viruslastkontrollen können ein Hinweis auf eine mangelnde Versorgung, insbesondere bei Hausärzten, sein. Folglich sollten die zukünftigen effektiveren Therapien in spezialisierten Zentren durchgeführt werden, damit diese teuren Therapien ihre Effizienz auch entfalten können.

\section{Konsequenz für Klinik und Praxis}

- Im Krankenhaus festgestellte Hepatitis-C-VirusInfektionen sollten in die ambulant geführten Patientenakten übernommen werden.

- Eine erstmals festgestellte Hepatitis C sollte nicht automatisch als akute HCV-Infektion interpretiert werden. Hier sollten die notwendigen differentialdiagnostischen Maßnahmen durchgeführt oder die Patienten an spezialisierte Zentren überwiesen werden.

- Die Diagnose „akute Hepatitis C“ sollte im Praxisverwaltungssystem nicht als Dauerdiagnose kodiert werden, sondern bei nicht erfolgreicher Therapie nach 6 Monaten in eine „chronische Hepatitis C“ geändert oder bei erfolgreicher Therapie mit dem Kürzel für „Zustand nach“ versehen werden.

- Die für einen Therapiebeginn und Therapieerfolg notwendigen Viruslastkontrollen sind durchzuführen oder die Patienten sind in spezialisierte Zentren zu überweisen.

\section{Literaturverzeichnis}

1 Management of hepatitis C: 2002. NIH Consens State Sci Statements 2002; 19: 1-46

2 Cornberg M, Höner zu Siederdissen C, Maasoumy B, Manns MP. [New direct-acting antiviral agents for the treatment of chronic hepatitis $C$ in 2014]. Internist (Berl) 2014; 55: 390-400

3 Di Bisceglie AM. Natural history of hepatitis C: its impact on clinical management. Hepatology 2000; 31: 1014-1018 
4 Höner zu Siederdissen C, Cornberg M. Neue Interferonfreie Therapien für Patienten mit chronischer Hepatitis C Genotyp 1. Dtsch Med Wochenschr 2014; 139: 2411-2414

5 Janssen Cilag International NV. Fachinformation. INCIVO $^{\circledR} 375$ mg Filmtabletten. 2011

6 Maasoumy B, Port K, Markova AA et al. Eligibility and safety of triple therapy for hepatitis C: lessons learned from the first experience in a real world setting. PLoS One 2013; 8: e55285

7 Merck Sharp \& Dohme Ltd. Fachinformation Victrelis $200 \mathrm{mg}$ Hartkapseln (Boceprevir). 2011

8 Offergeld R, Ritter S, Quabeck L et al. Infektionsepidemiologische Daten von Blutspendern in Deutschland 2007 [Epidemiological data on infections among blood donors in Germany 2007]. Bundesgesundheitsblatt Gesundheitsforschung Gesundheitsschutz 2010; 53: 1188-1196

9 Robert Koch-Institut. Zur Situation bei wichtigen Infektionskrankheiten in Deutschland Virushepatitis B, C und D im Jahr 2010. Epidemiologisches Bulletin 2011; 29: 14

10 Rockstroh JK, Bhagani S, Benhamou Y et al. European AIDS Clinical Society (EACS) guidelines for the clinical management and treatment of chronic hepatitis $B$ and C coinfection in HIV-infected adults. HIV med 2008; 9: 82-88

11 Santantonio T, Wiegand J, Gerlach JT. Acute hepatitis C: current status and remaining challenges. J Hep 2008; 49: 625-633

12 Sarrazin C, Berg T, Ross RS et al. Update der S3-Leitlinie Prophylaxe, Diagnostik und Therapie der Hepatitis-CVirus (HCV)-Infektion, AWMF-Register-Nr.: 021/012 [Prophylaxis, diagnosis and therapy of hepatitis $\mathrm{C}$ virus (HCV) infection: the German guidelines on the management of HCV infection]. Z Gastroenterol 2010; 48: 289-351
13 Sarrazin C, Berg T, Cornberg M et al. [Expert opinion on boceprevir- and telaprevir-based triple therapies of chronic hepatitis C]. Z Gastroenterol 2012; 50: 57-72

14 Schreier E, Radun D, Neuhauser H, Stark K. Hepatitis C. Gesundheitsberichterstattung des Bundes 2003; 15

15 Smith DB, Bukh J, Kuiken C et al. Expanded classification of hepatitis C Virus into 7 genotypes and 67 Subtypes: updated criteria and assignment web resource. Hepatology 2014; 59 : 318-327

16 Vermehren J, Schlosser B, Domke D et al. High prevalence of anti-HCV antibodies in two metropolitan emergency departments in Germany: a prospective screening analysis of 28. 809 patients. PLoS One 2012; 7: e41206

17 Villa E, Cammà C, Di Leo A et al. Peginterferon-A_2B plus ribavirin is more effective than peginterferon-A_2A plus ribavirin in menopausal women with chronic hepatitis C. J Viral Hepat. 2012; 19: 640-649

18 Wedemeyer H, Duberg AS, Buti M et al. Strategies to manage hepatitis $\mathrm{C}$ virus (HCV) disease burden. J Viral Hepat 2014; 21 Suppl 1: 60-89

\section{Danksagung}

Wir danken der SGH Consulting, Susanne Guthoff-Hagen und dem Team Gesundheit, Gesellschaft für Gesundheitsmanagement $\mathrm{mbH}$ für die Datenbankanalysen.

\section{Interessenkonflikt}

JT ist Mitarbeiter der Firma Janssen-Cilag GmbH. MC hat Honorare für Vorträge und Tätigkeiten in Advisory und Data Safety Boards erhalten von: Roche, MSD Sharp \& Dohme, Gilead Sciences, Janssen-Cilag, AbbVie, BristolMyers Squibb, Novartis
DOI 10.1055/s-0041-101101 Dtsch Med Wochenschr 2015; 140: e67-e73

(c) Georg Thieme Verlag KG . Stuttgart · New York . ISSN 0012-0472 